\title{
Frontal eye fields involved in shifting frame of reference within working memory for scenes
}

\author{
Mikkel Wallentin $^{\mathrm{a}, \mathrm{b}, *}$, Andreas Roepstorff ${ }^{\mathrm{a}, \mathrm{c}}$, Neil Burgess $\mathrm{d}, \mathrm{e}$ \\ ${ }^{a}$ Center for Functionally Integrative Neuroscience, Aarhus University Hospital, Nфrrebrogade, 8000 Aarhus C, Denmark \\ ${ }^{\mathrm{b}}$ Center for Semiotics, University of Aarhus, Denmark \\ c Institute for Anthropology, Aarcheology and Linguistics, University of Aarhus, Denmark \\ ${ }^{\mathrm{d}}$ Department of Anatomy, University College London, UK \\ ${ }^{\mathrm{e}}$ Institute of Cognitive Neuroscience, University College London, UK
}

Received 12 March 2007; received in revised form 18 August 2007; accepted 20 August 2007

Available online 25 August 2007

\begin{abstract}
Working memory (WM) evoked by linguistic cues for allocentric spatial and egocentric spatial aspects of a visual scene was investigated by correlating fMRI BOLD signal (or "activation") with performance on a spatial-relations task. Subjects indicated the relative positions of a person or object (referenced by the personal pronouns "he/she/it") in a previously shown image relative to either themselves (egocentric reference frame) or shifted to a reference frame anchored in another person or object in the image (allocentric reference frame), e.g. "Was he in front of you/her?" Good performers had both shorter response time and more correct responses than poor performers in both tasks. These behavioural variables were entered into a principal component analysis. The first component reflected generalised performance level. We found that the frontal eye fields (FEF), bilaterally, had a higher BOLD response during recall involving allocentric compared to egocentric spatial reference frames, and that this difference was larger in good performers than in poor performers as measured by the first behavioural principal component. The frontal eye fields may be used when subjects move their internal gaze during shifting reference frames in representational space. Analysis of actual eye movements in three subjects revealed no difference between egocentric and allocentric recall tasks where visual stimuli were also absent. Thus, the FEF machinery for directing eye movements may also be involved in changing reference frames within WM.
\end{abstract}

(C) 2007 Elsevier Ltd. All rights reserved.

Keywords: Allocentric reference frame; Egocentric reference frame; Space; Language; Working memory; Frontal eye fields; FEF; fMRI

\section{Introduction}

Contextual reference is a vital part of language function and cognition in general. Personal pronouns like "he/she/it/you" are among the most commonly used words in language (Leech, Rayson, \& Wilson, 2001). Such words, however, are only meaningful within the context of discourse. They are used for referencing elements in the perceptual surroundings and/or in memory. An important part of this referencing is an indication of

\footnotetext{
* Corresponding author at: Center for Functionally Integrative Neuroscience, Aarhus University Hospital, Nørrebrogade, 8000 Aarhus C, Denmark. Tel.: +45 89494380; fax: +45 89494400 .

E-mail address: mikkel@ pet.auh.dk (M. Wallentin).

URLs: http://www.cfin.au.dk (M. Wallentin), http://www.mikkelwallentin.dk (M. Wallentin).
}

which "frame of reference" (Kemmerer, 2006; Levinson, 2003) is used to "ground" the construction in a figure-ground relationship (Talmy, 2000), i.e. it makes a big difference whether somebody says "the tiger is in front of him" or "the tiger is in front of you". The frames of references used linguistically have, across languages, been shown to impact the way people construct spatial relations, also in non-linguistic contexts (e.g. Haun, Rapold, Call, Janzen, \& Levinson, 2006; Levinson, 2003). However, the neural underpinnings of this relationship is not well known.

In a recent paper (Wallentin, Roepstorff, Glover, \& Burgess, 2006) we showed how linguistically cued recall of spatial aspects of a recently viewed image (e.g. Fig. 1) compared to recall of nonspatial content (i.e. age of characters in the image) was supported by a network of brain regions known to be involved in spatial working memory without linguistic cueing (Smith \& 




Fig. 1. Example stimuli. Forty images like this were shown for $3000 \mathrm{~ms}$. Each image contained a man, a woman and a chair. Subjects were subsequently asked to recall the spatial relationship between these objects (allocentric reference frame), e.g. "Was he/she/it in front of him/her/it?" and the spatial relationship between an object and the subject (egocentric reference frame), e.g. "Was he/she/it in front of you?" Nonspatial relations were also probed (see Section 2) but are not covered in this paper (see Wallentin et al., 2006).

Jonides, 1998; Ungerleider, Courtney, \& Haxby, 1998). This network consisted mainly of posterior and medial parietal cortex, but also frontal eye fields (FEF). These findings were replicated in a purely linguistic study (Wallentin, Weed, Østergaard, Mouridsen, \& Roepstorff, in press) in which subjects recalled spatial aspects of a previously read sentence compared to recall of nonspatial information. This suggests that recall of linguistic and non-linguistic spatial information may rely on overlapping brain regions, i.e. comprehension of both types of information may occur via construction of a visuo-spatial mental model (Johnson-Laird, 1980; Lakoff \& Johnson, 1980; Talmy, 2000).

In the image recall experiment (Wallentin et al., 2006), the spatial condition actually consisted of two different spatial recall tasks: an egocentric recall task (e.g. "Was he/she/it in front of you?"), where subjects used their own frame of reference, i.e. the one given during encoding, as an anchor, and an allocentric recall task, in which subjects had to shift to a new frame of reference (e.g. "Was he/she/it in front of her/him/it?") relative to the orientation of a person or object in the encoded image. In studies of spatial long-term memory the distinction between the allocentric and egocentric reference frames has been vital (e.g. Burgess, Maguire, \& O'Keefe, 2002; O'Keefe \& Nadel, 1978), with hippocampus being involved predominantly in navigation tasks using an allocentric strategy whereas parietal or striatal regions have been found to be more involved in navigation using an egocentric strategy (Hartley, Maguire, Spiers, \& Burgess, 2003; Iaria, Petrides, Dagher, Pike, \& Bohbot, 2003).

Spatial working memory (Baddeley \& Hitch, 1974) and imagery supporting it may be inherently egocentric in nature (Byrne, Becker, \& Burgess, 2007; Milner, Djickerman, \& Carey, 1999; Schenk, 2006). Creating an actual image from an allocentric representation involves adopting a reference point and thereby transforms the representation into an egocentric framework. Parietal and prefrontal regions, such as the frontal eye fields, may play different roles in this process. Parietal areas may predominantly be associated with storage whereas prefrontal areas support control processes such as active maintenance or planning (Levy \& Goldman-Rakic, 2000; Oliveri et al., 2001; Shallice, 1988), using the posterior spatial representations as the source for these manipulations.

Adopting a certain frame of reference in working memory may involve brain regions responsible for updating viewpoint during perceptual processing, that is, regions involved in making or planning eye movements. In support of this notion, voluntary eye movements have been found to disrupt spatial working memory (Postle, Idzikowski, Sala, Logie, \& Baddeley, 2006). Conversely, in the absence of a visual stimulus regions such as FEF may contribute to reference frame shifts in representational space. This process may only be accompanied by simulated eye movements, e.g. motor intentions rather than actions (Colby \& Goldberg, 1999).

Few neuroimaging studies have been done on reference frame shifts in imagery and working memory, which have mainly been investigated in mental rotation tasks (e.g. Bonda, Petrides, Frey, \& Evans, 1995; Keehner, Guerin, Miller, Turk, \& Hegarty, 2006; Wraga, Shephard, Church, Inati, \& Kosslyn, 2005). Keehner et al. (2006) found a correlation in posterior parietal cortex between BOLD response and degrees of rotation in a mental rotation task, but no correlation with degrees of rotation in a perspective-shifting task. Adopting an allocentric frame of reference has been shown to activate a parieto-premotor network (e.g. Committeri et al., 2004; Galati et al., 2000; Schmidt et al., 2007; Vogeley et al., 2004; Zacks $\&$ Michelon, 2005). However, neither of these studies took performance into account, thereby making it impossible to rule out task-difficulty difference effects. Incorporating performance measures into a neuroimaging study of reference frame shifting and working memory was the aim of the present study.

Subjects were shown images like Fig. 1 and subsequently asked to recall the position of the woman, man and chair either relative to each other (allocentric frame of reference) or relative to the subject (egocentric frame of reference). Use of auditory language cues enabled us to investigate shifts in reference frames in a naturalistic way, mixing tasks seamlessly during scanning without the use of visual stimuli that would otherwise produce actual eye movements and thus introduce confounding activations in our regions of interest.

We predicted that fronto-parietal regions such as precuneus and FEF, both found to be more involved in spatial recall than in nonspatial recall (Wallentin et al., 2006; Wallentin et al., in press), would differentiate in their involvement during recall of spatial information when using allocentric and egocentric reference frames. Further, we hypothesised a hippocampal differentiation resembling that seen in navigation tasks (e.g. Burgess et al., 2002). This differentiation should be detectable in a contrastive analysis in which performance is taken into account. 


\section{Methods}

\subsection{Stimuli}

Forty images like Fig. 1 were constructed using Poser 5 software (Curious Labs, Santa Cruz, USA), each containing a man ("he/him"), a woman ("she/her"), and a chair ("it/it").

Four types of question sentences each asked subjects to recall specific information about the image:

- Spatial allocentric information: "Was he/she/it in front of him/her/it?"

- Spatial egocentric information: "Was he/she/it in front of you?"

- Nonspatial allocentric information: "Was he/she/it older than him/her/it?"

- Nonspatial egocentric information: "Was he/she/it older than you?"

Question sentences were delivered verbally to avoid primary perceptual overlap with the image stimuli (see Section 1). Sentences were recorded in a neutral female voice, and played through the standard pneumatic headphones of the scanner.

All sentences contained the same number of syllables and had exactly the same duration $(2000 \mathrm{~ms})$.

This paper deals mainly with results related to the AlloSpace-EgoSpace dichotomy. A detailed account of the Space-NonSpace contrast has been presented elsewhere (Wallentin et al., 2006).

\subsection{Experimental design}

Forty event sequences (each $30 \mathrm{~s}$ ) made up the experiment. These sequences included the display of a visual image and four subsequent, verbally presented questions about the contents of the image.

Images were shown for $3000 \mathrm{~ms}$, after which a white fixation-cross appeared in the middle of the screen. After a short, semi-random delay (1000-3000 ms, mean $2000 \mathrm{~ms}$ ), the four $2000 \mathrm{~ms}$ questions were played with $4000-8000 \mathrm{~ms}$ semi-randomised intervals (mean inter-stimulus interval $6000 \mathrm{~ms}$ ). The order of the four types of questions in the sequence was counterbalanced across events. The randomisation of intervals and order was made to ensure orthogonal response functions for analysis (see e.g. Henson, 2006).

Subjects were first instructed about the contents of the experiment by the experimenter, and a written instruction was then read to them, to ensure that all had received the same information. They were asked to look at the image and to try to remember the content. Due to the relatively small projection screen, subjects were asked to consider objects in the middle of the image as in front of themselves, and objects in the periphery as not. The instructions for the egocentric questions read: "One question type asks if a character or object was in front of you. You should try to remember the place of the object in question. If the object was somewhere in the middle of the screen, you should consider it as in front of you, and answer 'yes'. If the object was placed far out on one of the sides, you should answer 'no' to the question." The instructions for the allocentric questions read: "The second type of question asks you about the spatial relationship between the objects, e.g. 'Was he in front of her?' In this case you should try to remember the orientation of the woman, and try to remember, whether the man was standing in front of her, seen from her perspective, not your own."

Subjects were told not to rehearse possible questions during encoding. For each sentence they were asked to respond "yes/no" by button press as quickly and accurately as possible immediately after hearing the question, with either right index finger or right middle finger.

In order to secure a full understanding of the paradigm, subjects were submitted to a pre-run of two event cycles (two times an image + four questions) like those in the experiment prior to scanning.

\subsection{Subjects}

Twenty one healthy volunteers ( 9 female, 12 male), mean age $22.2 \pm 1$ (S.E.M.) years, participated in the experiment. All subjects were right-handed, and reported having English as their primary language. All participants gave informed, written consent in accordance with requirements of the local medical ethics committee. Subjects were paid $15 £$ for their participation. One subject was subsequently excluded from the study due to performance not exceeding chance level on the spatial task.

\subsection{Scanning parameters}

Functional images were acquired on a 3-T MR system with a standard head coil (Siemens Allegra, Erlangen, Germany). Four hundred and seventy contiguous multislice $\mathrm{T} 2 *$-weighted images were obtained using an echo planar imaging sequence with the following parameters: repetition time (TR): $2600 \mathrm{~ms}$, echo time (TE): $30 \mathrm{~ms}$, flip angle: $90^{\circ}$. Forty sequential, descending $2 \mathrm{~mm}$ axial slices (with $1 \mathrm{~mm}$ gap between slices) were obtained per volume, with an inplane resolution of $3 \mathrm{~mm} \times 3 \mathrm{~mm}$. The first five images from each session were discarded from the analysis due to burn in T1 effects.

\subsection{Data analysis}

Raw data were reconstructed and converted into Analyze format, spatially realigned (Friston et al., 1995a), unwarped according to all six motion parameters (Andersson, Hutton, Ashburner, Turner, \& Friston, 2001), slice time corrected, and normalised (Ashburner \& Friston, 1999) to the MNI template using SPM2 (Statistical Parametric Mapping, Wellcome Department of Imaging Neuroscience, University College London, London, UK; http://www.fil.ion.ucl.ac.uk) executed in MATLAB (Mathworks Inc., Sherborn, Massachusetts, USA). After normalization, the images were smoothed with a 10 -mm full-width at halfmaximum Gaussian filter.

A design matrix was made, including a regressor with onsets for all images (duration $3000 \mathrm{~ms}$ ) and separate regressors for each sentence type (duration $2000 \mathrm{~ms}$ ). A parametric modulation regressor was included for each sentence type, using response-time measurements as modulation parameters. Lastly, a "Button Press" regressor, including all button-press measurements was included to model activation related to motor output. All events were modelled using the standard hemodynamic response function of SPM2. Neither the parametric regressors nor the "button press" regressor were used in the present analysis (but see Wallentin et al., 2006).

The model fit to the data was estimated for each participant, using a general linear model (Friston, Holmes et al., 1995) with a 128-s high-pass filter, and AR(1) modelling of serial correlation. Two contrasts were created from the estimated $\beta$ weights for each subject, one related to the effect difference between spatial and nonspatial recall ([AlloSpace +EgoSpace][AlloNonSpace + EgoNonSpace]), and one related to recall of allocentric space versus egocentric space (AlloSpace-EgoSpace). These were used in two separate second-level random effects analyses in order to facilitate inferences about population effects (Friston, Holmes, Price, Buchel, \& Worsley, 1999). Significance threshold was set to $P<0.05$, false discovery rate (FDR) corrected for multiple comparisons. A third second-level analysis was conducted on the $\beta$ estimates for the AlloSpace regressor (i.e. the AlloSpace-Baseline contrast). The Space-NonSpace contrast was analysed with a one-sample $t$-test in SPM2 (detailed results from this are reported in Wallentin et al., 2006). The random effects analysis of the AlloSpace-EgoSpace contrast included both a constant main effects regressor, and a performance regressor (see below). Performance effects were masked to include only regions found to be significantly different both in the Space-NonSpace second level-test, the AlloSpace-Baseline second level-test, and the AlloSpace-EgoSpace main effects contrast. Significance threshold for the performance-related analysis was set to $P<0.05$ (FDR corrected for multiple comparisons). We only took into consideration regions were good performers have a higher response compared to poor performers.

In order to investigate performance effects across subjects and spatial tasks, summary scores (i.e. average response time and percentage correct responses) for each subject from the AlloSpace and EgoSpace conditions were $z$-score normalised and subjected to a principal component analysis (PCA) in Matlab. Only results from the first component are considered here. Subject scores from the first component were entered into a second-level regression analysis on the AlloSpace-EgoSpace contrast using SPM2. A similar PCA analysis was conducted on behavioural data from both the allocentric spatial task alone and from the egocentric spatial task alone (i.e. average response time and accuracy). The first component from each individual task-analysis was used to investigate per- 
formance correlations with voxel-data from the AlloSpace task versus baseline and from the EgoSpace versus baseline.

\subsection{Eye tracking}

To give an indication of whether differences in eye movement patterns contributed to differences seen in scanning contrasts, we conducted eye tracking in three subjects during scanning. Eye tracking was conducted with an ASL E5000 system (http://www.a-s-1.com) with E504 analysis control box and Long Range Optics camera. Due to time limitations, the eye tracking procedure was discontinued after scanning the first three subjects.

We compared eye movements for the encoding condition and the two spatial recall conditions. Pupil location was measured continuously with a $60 \mathrm{~Hz}$ frequency. 3000 milliseconds after the onset of each event, squared location differences from sample to sample (along the horizontal and vertical axes individually) were summed in MatLab to give a measure of the total pupil movement during the event. Movement across the 40 events between conditions was compared for each of the three subjects using a Kruskal-Wallis test of equal medians in Matlab.

\section{Results}

\subsection{Behavioural data}

Subjects responded both faster and more accurately during recall with egocentric reference frame (mean RT: $2832 \pm 74 \mathrm{~ms}$ (S.E.M.); mean \% correct responses: $85 \pm 2 \%$ (S.E.M.)) than during recall with allocentric reference frame (mean RT: $3188 \pm 75 \mathrm{~ms}$ (S.E.M.); mean \% correct responses: $79 \pm 2 \%$ (S.E.M.)). Both the difference between RTs for allospace and egospace $(t(1,19)=9.79, P<0.0000001)$ and the difference in accuracy between the two tasks $(t(1,19)=-4.05, P<0.0001)$ were statistically highly significant when performance was compared using paired $t$-tests.

Subject performance was highly correlated between the two spatial conditions, both in terms of response time $(r=0.89$, $P<0.0001)$ and in terms of percent correct responses $(r=0.7$, $P=0.0006)$. This suggests that subjects who performed well on the allocentric task also performed well on the egocentric task, in spite of the task-difficulty difference between the two tasks.

To find a common performance measure spanning both the response time and the accuracy measurements across the two conditions, we subjected these four measures to a principal component analysis. The first component weighted the four measures roughly equal (coefficients: RT_AlloSpace: -0.52; RT_EgoSpace: -0.52; \%Cor_AlloSpace: 0.42; \%Cor_EgoSpace: 0.52). Subjects who scored high on the first component were good overall performers (low RT and high accuracy) while subjects with a low score on the first PCA component were poor overall performers. The first PCA component explained $72 \%$ of the variance in the behavioural data.

In order to be able to investigate performance on the individual spatial recall tasks we also conducted a PCA on RT and accuracy for each of these tasks. Again it was found that the first component weighted the two inputs equally (coefficients for first component of AlloSpace PCA: RT_AlloSpace: -0.71; \%Cor_AlloSpace: 0.71 ; coefficients for first component of EgoSpace PCA: RT_EgoSpace: -0.71; \%Cor_EgoSpace: 0.71). Again subjects with a high score on the first component of these two analyses were overall good performers on the task (low RT and high accuracy).

In each of the three subjects for whom we monitored eye movements during scanning, we found significantly more eye movement during encoding-i.e. during viewing of the images compared to either of the two spatial recall conditions, predominantly along the horizontal axis $(P<0.00001)$, but also along the vertical axis $(P<0.05)$. We found no difference in eye movements between the two spatial recall conditions $(P>0.05)$ in any of the three subjects. In terms of performance, these three subjects were representative of the whole group (placed 3rd, 6th and 18th in the overall PCA measure of performance). We thus have no reason to believe that spatial recall contrast differences in the fMRI results were driven by overt eye movement differences.

\subsection{Scanning data}

A number of regions exhibited a significantly higher response $(P<0.05$, FDR-corrected) during spatial recall (Fig. 2A, see Wallentin et al., 2006 for details). Among these were frontal eye fields MNI [-24, 4, 62] and [28, 2, 54]. A smaller number of regions exhibited greater response during allocentric spatial recall than during egocentric spatial recall, including among others the frontal eye fields (FEF), bilaterally: MNI $[-36,2,50]$ and [38, 4, 46]) (Fig. 2B). This contrast, however, is confounded with task difficulty differences (see above), and therefore it cannot stand alone. To confront this problem we also looked at regions significantly activated by allocentric spatial recall relative to baseline (Fig. 2C). Again we found peaks in the FEF region: MNI $[-30,-2,64]$ and $[24,14,50]$. For the use in further explorations we created a mask that included only regions significant in all three contrasts above (Fig. 2D).

Within the regions defined by our mask we searched for clusters that correlated with our performance measure. When correlating results from the Allospace-EgoSpace contrast within

Table 1

Regions with a significant correlation $(P<0.05$, FDR-corrected) between performance on the spatial tasks and contrast difference between allocentric and egocentric spatial recall, masked to only include regions significant in Space-NonSpace, AlloSpace-EgoSpace and AlloSpace-Baseline contrasts

\begin{tabular}{|c|c|c|c|}
\hline Contrast & Putative anatomical region & Peak MNI & $z$-Score \\
\hline & $\mathrm{R}$ front. eye field-BA 6 & {$[28,10,54]$} & 3.85 \\
\hline & L inf. parietal lobule-BA 40 & {$[-32,-50,40]$} & 3.75 \\
\hline & L sup. parietal lobule-BA 7 & {$[-20,-72,56]$} & 2.14 \\
\hline & $\mathrm{R}$ precuneus-BA 7 & {$[8,-60,46]$} & 3.95 \\
\hline & L inf. front. operculum (Broca's)—BA 44 & {$[-52,12,22]$} & 2.24 \\
\hline
\end{tabular}




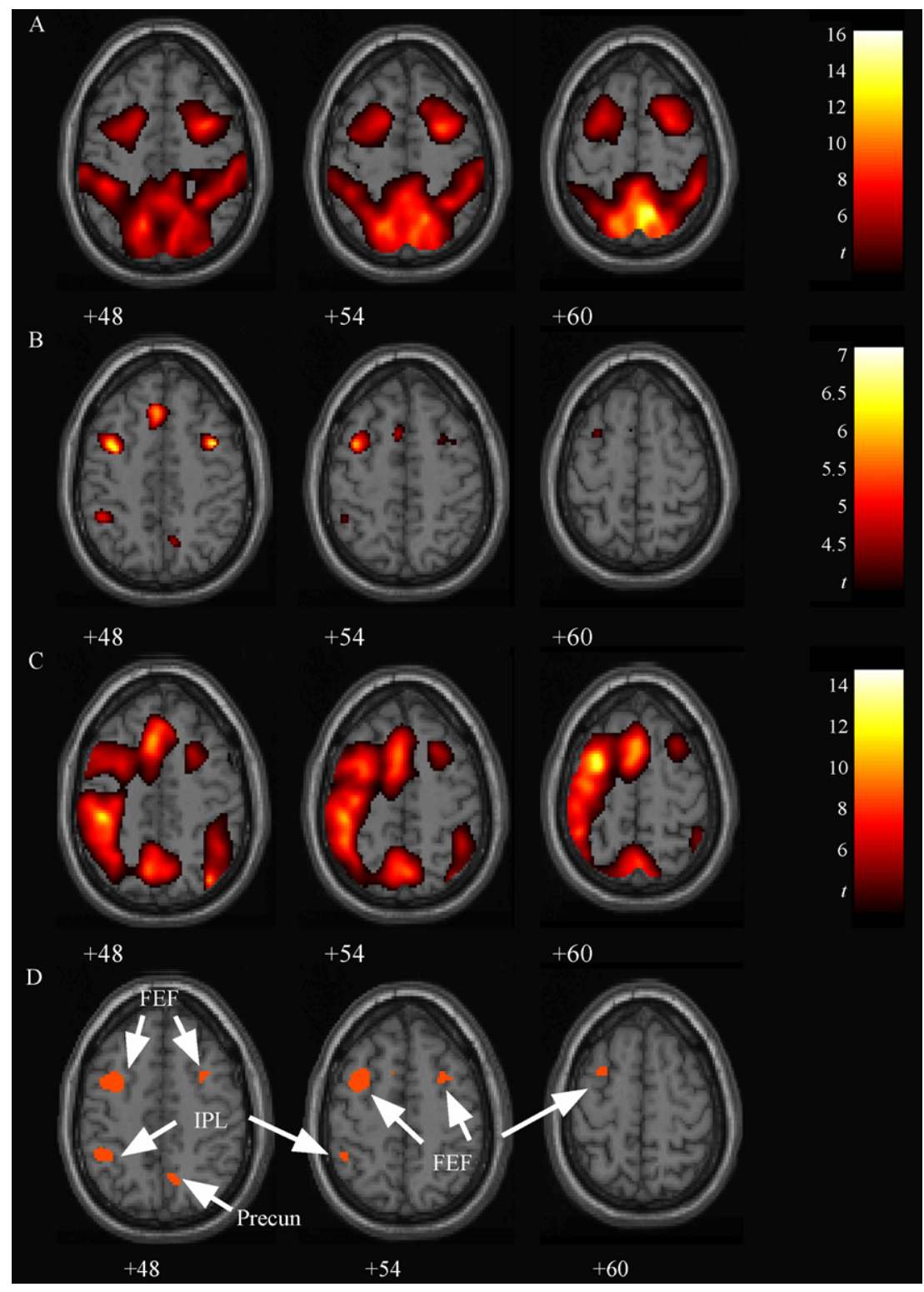

Fig. 2. Main contrasts and overlaps. (A) Space $>$ NonSpace. A large network of brain regions, including FEF had a significantly higher BOLD response $(P<0.05$, FDR corrected) during recall of spatial content recall (e.g. "Was he in front of her?") relative to recall of nonspatial content (e.g. "Was he older than her/you?"). See Wallentin et al. (2006) for further details. (B) AlloSpace > EgoSpace. Frontal eye fields were again among the regions where subjects had a significantly higher response during allocentric spatial recall (e.g. "Was he in front of her?") than during egocentric spatial recall (e.g. "Was he in front of you?") at $P<0.05$, FDR corrected. This contrast, however, is confounded with task difficulty, and cannot stand alone. (C) AlloSpace $>$ Baseline $(P<0.05$, FDR corrected). Again, we see a large network of regions activated above baseline during recall using allocentric spatial reference frames. These networks not only include motor regions for button presses, but also FEF and precuneus. (D) Overlapping regions. A small number of regions were found in all three analyses above. These regions included precuneus (Precun), inferior parietal lobule (IPL) and frontal eye fields (FEF). These regions were used as an inclusive mask for analyses of performance related effects.

our mask with performance level (first behavioural PCA component), we found a significant bilateral $(P<0.05$, FDR-corrected $)$ FEF correlation (left FEF, $z$-score: 3.90, MNI: $[-26,8,60]$; right FEF: $z$-score: 3.85 , peak MNI: $[28,10,54]$ ) (Fig. 3, Table 1), along with correlations in left inferior frontal operculum (Broca's): $z$-score: 2.24, peak MNI: $[-52,12,22]$, precuneus: $z$-score 3.95 , peak MNI: $[8,-60,46]$; left superior parietal lobule: $z$-score: 2.14 , peak MNI: $[-20,-72,56]$; left inferior parietal lobule: $z$-score: 3.75 , peak MNI: $[-32,-50$, 40]. Thus, compared to poor performers, good performers had a greater BOLD response difference between Allospace and
Egospace conditions in FEF, Broca's region and three regions in parietal cortex. To further constrain our interpretation we checked if it could be determined whether this correlation was linked to the allocentric or the egocentric spatial condition. For this purpose we examined the correlation between performance for the individual tasks and the individual condition responses at the peak correlation voxels (Table 1). We found that in both left FEF $(r=0.60, P=0.006)$ and right FEF $(r=0.45, P=0.04)$ allocentric spatial recall yielded a significantly greater response in good performers than in poor performers (Fig. 3). This was not the case for Broca's region $(r=0.23, P=0.33)$, precuneus 

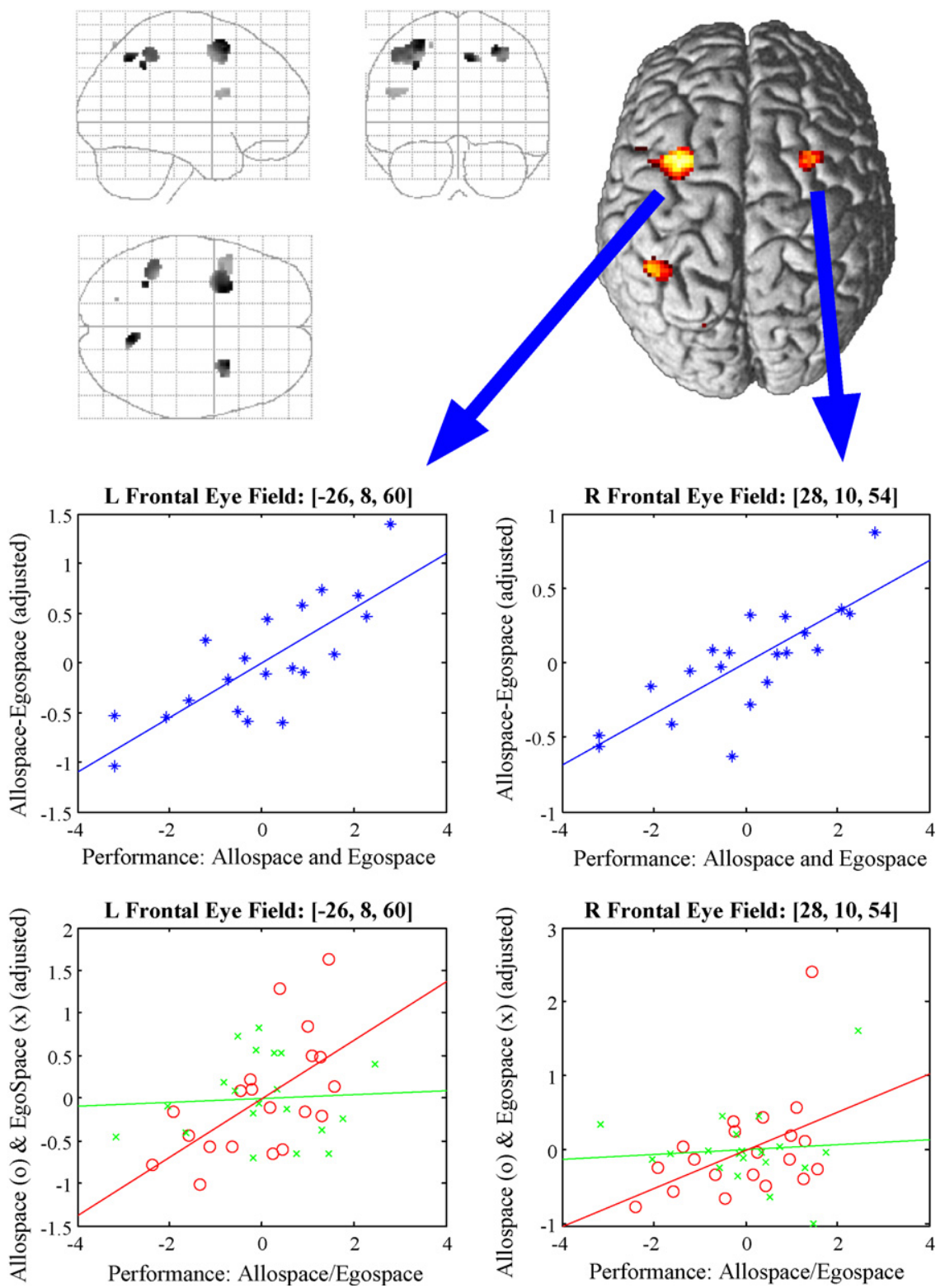

Fig. 3. Frontal eye field activation-performance correlation. (Top) Glass brain shows regions where good performers had a higher response difference between allocentric spatial recall and egocentric spatial recall compared to poor performers $(P<0.05$, FDR corrected, when masked to only include regions significant in the both the Space-NonSpace contrast, the AlloSpace-EgoSpace contrast and the AlloSpace-Baseline contrast). (Middle) The response difference in frontal eye fields (FEF), bilaterally, was strongly correlated with performance level as measured by the first component of a principal component analysis combining response time and $\%$ correct responses for both allocentric and egocentric spatial tasks. Higher score on the first PCA comp. means better performance. Good performers had a greater response difference than poor performers: AlloSpace-EgoSpace vs. first PCA comp.: L FEF: $r=0.79, P=0.00003$; R FEF: $r=0.78, P=0.00005$. (Bottom) When looking at performance and fMRI response from the different conditions individually, we found that response for allocentric recall $(\bigcirc)$ was significantly correlated with performance on allocentric tasks, whereas response for egocentric recall (x) was not correlated with performance. Again, performance was found by submitting RT and accuracy from an individual tasks to a PCA analysis. Higher score means better performance: L FEF: AlloSpace vs. first PCA comp. (allocentric RT and accuracy): $r=0.60, P=0.006$; EgoSpace vs. first PCA comp. (egocentric RT and accuracy): $r=0.06, P=0.79$; R FEF: AlloSpace vs. first PCA comp.: $r=0.45, P=0.04$; EgoSpace vs. first PCA comp.: $r=0.09, P=0.71$. This clearly demonstrates that the frontal eye fields are involved in effectively solving the task of reconstructing allocentric spatial relations.

$(r=0.34, P=0.15)$, SPL $(r=0.06, P=0.8)$ or IPL $(r=0.33$, $P=0.15)$, and it was not the case for egocentric spatial recall in any region either (Fig. 3). Thus, we have several indicators that FEF plays a role in shifting to an allocentric spatial reference frame within a recently experienced visual scene. These findings further support the notion that this effect is in fact linked to the allocentric spatial recall task and not to relative changes in the control tasks or in the baseline (Gusnard \& Raichle, 2001).

\subsubsection{Egocentric spatial recall}

When looking for regions involved in egocentric spatial recall we found no regions that were both more activated in the Space-NonSpace contrast, the EgoSpace-AlloSpace con- 
trast with a performance correlation. Due to the limitations of this study in terms of our task difficulty confound, we cannot say anything about the use of egocentric spatial recall without having a very strong indication of performance correlates in the imaging signal.

\section{Discussion}

We investigated differences in fMRI BOLD response from recalling spatial aspects of a visual scene (e.g. Fig. 1) seen with an allocentric frame of reference (i.e. "Was he in front of her?") compared to recalling it seen from the subject's own egocentric frame of reference (i.e. "Was he in front of you?").

To confront the problems of task difficulty differences inherent between these types of tasks we looked for regions where the BOLD-response could be shown to have a strong correlation with between-subjects behaviour. We created a mask with regions of interest. These regions consisted of voxels that showed up significant in both the Space-NonSpace, the AlloSpace-EgoSpace and the AlloSpace-Baseline contrasts (Fig. 2). Within these regions we found six regions where overall performance on the two spatial tasks correlated significantly with the AlloSpace-EgoSpace contrast (Table 1) in such a way that good performers had a greater response difference than poor performers. To further validate these findings we investigated whether the AlloSpace parameter estimates (i.e. AlloSpace-Baseline) in peak voxels from these six regions correlated with performance on the allocentric spatial recall task. This was found in two regions only, the frontal eye fields, bilaterally (Fig. 3). In the following we will focus our discussion on these two regions, where results were robust through all our analyses. Subsequently, we briefly discuss our hypotheses regarding precuneus and hippocampus.

\subsection{Frontal eye fields (FEF)}

The coordinates for which the correlations with performance are observed (Table 1, Fig. 2) overlap with FEF as located by use of electrical stimulation for evoking eye movements (Blanke et al., 2000).

FEF are known to be involved in eye movements and in both overt and covert shifting of spatial attention to present stimuli (see Corbetta \& Shulman, 2002; Pessoa, Kastner, \& Ungerleider, 2003 for reviews). However, we did not see any differences in eye movements in three representative subjects, and the FEF response observed in our study occurred while there was no visual stimulus present apart from a fixation cross in the middle of the projection screen. Therefore, even if eye movement differences had been present during recall, these would have to be considered a consequence of the image recall task rather than a precursor. Further, the encoded image was the same for both allocentric and egocentric tasks. Therefore, the neural response in FEF must be linked to the manner in which the image was reconstructed during allocentric recall rather than the content of what was recalled. We interpret this as indicating that shifting reference frames may involve mock eye movements in representational space (Burgess, Becker, King, \& O'Keefe, 2001; Byrne et al., 2007) whereas recalling a scene from one's own reference point may not.

The frontal eye fields have been seen to respond more to voluntary shifts of attention than stimulus driven shifts (Kincade, Abrams, Astafiev, Shulman, \& Corbetta, 2005) and also to so called "retrospective cues", i.e. cues referring to a previous visual image held in short-term memory (Nobre et al., 2004), and level of activity during the maintenance period of a working memory task has been shown to predict performance (Pessoa, Gutierrez, Bandettini, \& Ungerleider, 2002). This study, however, is the first to show a direct link between performance during reference frame shifts and level of frontal eye field BOLD response in a task where subjects are tested on both allo- and egocentric conditions with the same encoding stimulus, thus keeping the maintenance period activity constant.

In a spatial WM study on six subjects, Rowe et al. (2000) found FEF activation ([-22, 8, 60] and [24, 4, 54]) for the maintenance period but not for retrieval. Due to differences in duration between maintenance and retrieval and the natural time lock between them, a direct comparison is difficult (and indeed not conducted by Rowe et al.). In our study, the maintenance period is represented in the unmodelled baseline, and relative to this we see a significant increase in activity during spatial recall with shifts in frame of references (see Fig. 2C), and this effect is larger than with egocentric recall (Fig. 2B). Our analysis cannot be used to say anything about the maintenance period itself, but since no shifts in reference frames were required during retrieval in the Rowe et al. study this, along with their small subject group, may explain why they do not se activation during recall. Our results may thus be compatible with the notion that FEF play a role during spatial WM maintenance, but add to this a role in shifting frame of reference.

Wraga et al. (2005) found left FEF activation in a study of both imagined object rotations [-36, 2, 48] and self-rotation $[-32,10,44]$. Object rotation had a significantly higher activation than self-rotation, but at a more posterior location $[-32$, $-6,43]$ than the one observed in our study. Galati et al. (2000) found activation in left FEF $[-28,8,52]$ during both an egocentric and an allocentric judgement task, but this activation was higher in the egocentric than in the allocentric. In this study, subjects judged, in the egocentric task, whether a movable vertical bar was placed to the left or to the right of the subject's midline and, in the allocentric task, relative to the middle of a longer, also movable, horizontal bar. Using a similar paradigm, however, Neggers et al. (2006) notice a significant interaction between the task-irrelevant allocentric cue and behavioural outcome in the egocentric condition, thus indicating that allocentric processing may be influencing the egocentric results obtained in these kinds of studies. Committeri et al. (2004) show that spatial judgements relative to a stable landmark have the highest response in FEF, but with similar activation across observer-relative and objectcentred judgements (no significant difference on a single-voxel level). Since landmark-relative judgements may require shifts in reference frames just like object-relative judgements, these results are not inconsistent with our own. Similarly, Vogeley et al. (2004) found higher activation in left FEF [-30, 0, 52] during an allocentric judgement task relative to an egocentric 
task, and David et al. (2006) found the same in right FEF [38, $2,54]$. As with our study, the contrasts in these studies are confounded with task-difficulty differences, but, unlike our study their relatively small number of subjects $(n=11$ and 13) makes it difficult to assess performance-related variation. Furthermore, since all of these studies involve online spatial judgements, viewpoint shifts and eye movements naturally interact and are to a large degree inseparable. By using a short-term memory paradigm with auditory cues, the present study avoids this problem.

In summary: Spatial recall yields more activation than nonspatial recall in FEF (Fig. 2A); recall of allocentric spatial information from imagery yields more activation than egocentric spatial recall in FEF, bilaterally (Fig. 2B). Allocentric spatial recall also yields activation in FEF relative to baseline (Fig. 2C). On top of this we have a strong performance correlation with BOLD response across subjects. The fact that good subjects had a greater response difference than poor performers rules out task difficulty effects, since good performers presumably find the task easier to solve than poor performers.

A possible caveat, however, is that the allocentric task involves comparing two objects in representational space, whereas the egocentric task involves evaluating the position of only one object. The FEF activity difference between the two tasks may thus be thought to reflect the number of virtual eye movements needed to evaluate more objects. The correlation with performance level, however, suggests that the difference between allocentric and egocentric inference cannot be understood as simply a difference in the total number of virtual eye movements or attention shifts necessary to complete the task. Such an interpretation would imply that good performers had more attention shifts during the allocentric task than poor performers. This speaks counter to the fact that the same subjects had shorter response times.

Our findings are compatible with patient studies showing that FEF lesions have an effect on spatial short-term memory but not on saccade inhibition (Gaymard, Ploner, Rivaud-Pechoux, \& Pierrot-Deseilligny, 1999). That is, our observed FEF activation cannot be said to reflect increased saccadic inhibition due to task difficulty differences. This is further supported by behavioural studies showing a selective disruption of spatial working memory by voluntary eye movements (Postle et al., 2006). If FEF are involved in both processes, one may interfere with the other, whereas other distracters, such as words, do not have a disruptive effect. Taken together, this leads to a prediction that voluntary eye movements should have less disruptive effects on spatial WM with an egocentric content than on spatial WM with an allocentric content. Further research is needed to investigate this hypothesis.

\subsection{Posterior parietal cortex}

We also found significant correlations between performance and the AlloSpace-EgoSpace contrast in dorsoposterior parietal cortex where the main focus of the Space-NonSpace recall dichotomy was located (Wallentin et al., 2006; Wallentin et al., in press). We found correlations in both inferior and superior pari- etal lobules along with precuneus. These correlations, however, could not be shown to originate from a variation in the allocentric spatial recall task alone, since we found no correlation between performance and the AlloSpace-Baseline contrast. Due to the limitations of our design, we therefore report this result with great caution. Further studies are needed to show if some or all of these results can be replicated.

\subsection{Hippocampus}

The hippocampus is known to play a vital role in the processing of allocentric spatial maps (O'Keefe \& Nadel, 1978). But while the hippocampus has repeatedly been shown to be involved in remembering/processing allocentric representations in an environmental framework, in particular relative to large/stable boundaries and landmarks (e.g. Doeller, King, \& Burgess, 2006; O'Keefe \& Burgess, 1996), our lack of hippocampal activation, we speculate, may indicate that it is not necessarily involved in reconstructing short-lived spatial relations (i.e. those existing between movable objects or people) be it relative to nonspatial relations (Wallentin et al., 2006) or relative to egocentric spatial relations. We acknowledge that our data only supply quite weak support for such a claim, but it is consistent with place cell results in rats, in which place cell firing has been shown to be controlled by large-scale geometric determinants (O'Keefe \& Burgess, 1996), but not by smaller "landmarks" placed inside the experimental arena (Cressant, Muller, \& Poucet, 1997). Rather, processing spatial relations that are centred on less-stable objects may depend on parietal, striatal or prefrontal areas (Burgess, Jeffery, \& O'Keefe, 1999; Doeller et al., 2006; Ungerleider et al., 1998; Wallentin et al., 2006) that cooperate with the medial temporal lobe only if the spatial relations become encoded into long-term memory (Burgess et al., 2001).

\section{Conclusion}

We have shown a linear relationship between performance on a task in which subjects recalled an image seen from an allocentric reference point and BOLD response in frontal eye fields (FEF). Good performers had higher activity than poor performers in this region relative to both an egocentric recall task and relative to fixation baseline. A main effects analysis also showed that FEF were more activated overall across subjects during allocentric spatial recall than during egocentric spatial recall. We conclude that FEF are involved in shifting to an allocentric frame of reference in imagery of recently viewed images, consistent with a role for mock motor efference signals in directing attention within imagery (Burgess et al., 2001; Byrne et al., 2007).

\section{Acknowledgements}

This work was supported by an EU NEST grant on "Wayfinding", the Medical Research Council, U.K., The Faculty of Humanities at University of Aarhus, and by The Danish National Research Foundation's grant to Center for Functionally Integrative Neuroscience. The authors wish to thank Rebecca Glover for 
help with data acquisition, John O'Keefe, Christian F. Doeller, Tom Hartley, John King, Torben Ellegaard Lund, Kim Mouridsen and Ethan Weed for their kind help and expertise during the course of this experiment.

\section{References}

Andersson, J. L. R., Hutton, C., Ashburner, J., Turner, R., \& Friston, K. (2001). Modeling geometric deformations in EPI time series. NeuroImage, 13(5), 903-919.

Ashburner, J., \& Friston, K. J. (1999). Nonlinear spatial normalization using basis functions. Human Brain Mapping, 7(4), 254-266.

Baddeley, A. D., \& Hitch, G. J. (1974). Working memory. In G. A. Bower (Ed.), Recent advances in learning and motivation (Vol. 8, pp. 47-90). New York: Academic Press.

Blanke, O., Spinelli, L., Thut, G., Michel, C. M., Perrig, S., Landis, T., et al. (2000). Location of the human frontal eye field as defined by electrical cortical stimulation: Anatomical, functional and electrophysiological characteristics. NeuroReport, 11(9), 1907-1913.

Bonda, E., Petrides, M., Frey, S., \& Evans, A. (1995). Neural correlates of mental transformations of the body-in-space. Proceedings of The National Academy of Sciences of The United States of America, 92(24), 11180-11184.

Burgess, N., Becker, S., King, J. A., \& O'Keefe, J. (2001). Memory for events and their spatial context: Models and experiments. Philosophical Transactions of The Royal Society of London, Series B, Biological Sciences, 356(1413), 1493-1503.

Burgess, N., Jeffery, K. J., \& O'Keefe, J. (1999). Integrating hippocampal and parietal functions: A spatial point of view. In N. Burgess, K. J. Jeffery, \& J. O'Keefe (Eds.), The hippocampal and parietal foundations of spatial cognition (pp. 3-29). Oxford University Press.

Burgess, N., Maguire, E. A., \& O'Keefe, J. (2002). The human hippocampus and spatial and episodic memory. Neuron, 35, 625-641.

Byrne, P., Becker, S., \& Burgess, N. (2007). Remembering the past and imagining the future: A neural model of spatial memory and imagery. Psychological Review, 114(2), 340-375.

Colby, C. L., \& Goldberg, M. E. (1999). Space and attention in parietal cortex. Annual Review of Neuroscience, 22, 319-349.

Committeri, G., Galati, G., Paradis, A.-L., Pizzamiglio, L., Berthoz, A., \& LeBihan, D. (2004). Reference frames for spatial cognition: Different brain areas are involved in viewer-, object-, and landmark-centered judgments about object location. Journal of Cognitive Neuroscience, 16(9), 1517-1535.

Corbetta, M., \& Shulman, G. L. (2002). Control of goal-directed and stimulusdriven attention in the brain. Nature Reviews. Neuroscience, 3(3), 201-215.

Cressant, A., Muller, R. U., \& Poucet, B. (1997). Failure of centrally placed objects to control the firing fields of hippocampal place cells. The Journal of Neuroscience: The Official Journal of The Society for Neuroscience, 17(7), 2531-2542.

David, N., Bewernick, B. H., Cohen, M. X., Newen, A., Lux, S., Fink, G. R., et al. (2006). Neural representations of self versus other: Visual-spatial perspective taking and agency in a virtual ball-tossing game. Journal of Cognitive Neuroscience, 18(6), 898-910.

Doeller, C. F., King, J. A., \& Burgess, N. (2006). Separation of hippocampal versus striatal memory systems for locations defined by boundaries versus single landmarks. NeuroImage, 31(supplement 1), S58.

Friston, K. J., Ashburner, J., Poline, J. B., Frith, C. D., Heather, J. D., \& Frackowiak, R. S. (1995). Spatial registration and normalization of images. Human Brain Mapping, 2, 165-189.

Friston, K. J., Holmes, A. P., Price, C. J., Buchel, C., \& Worsley, K. J. (1999). Multisubject fMRI studies and conjunction analyses. NeuroImage, 10(4), 385-396.

Friston, K. J., Holmes, A. P., Worsley, K. J., Poline, J. B., Frith, C. D., \& Frackowiak, R. S. (1995). Statistical parametric maps in functional imaging: A general linear approach. Human Brain Mapping, 2, 189-210.

Galati, G., Lobel, E., Vallar, G., Berthoz, A., Pizzamiglio, L., \& Le Bihan, D. (2000). The neural basis of egocentric and allocentric coding of space in humans: A functional magnetic resonance study. Experimental Brain Research, 133(2), 156-164.
Gaymard, B., Ploner, C. J., Rivaud-Pechoux, S., \& Pierrot-Deseilligny, C. (1999). The frontal eye field is involved in spatial short-term memory but not in reflexive saccade inhibition. Experimental Brain Research, 129(2), 288-301.

Gusnard, D. A., \& Raichle, M. E. (2001). Searching for a baseline: Functional imaging and the resting human brain. Nature Reviews. Neuroscience, 2(10), 685-694.

Hartley, T., Maguire, E. A., Spiers, H. J., \& Burgess, N. (2003). The well-worn route and the path less traveled: Distinct neural bases of route following and wayfinding in humans. Neuron, 37(5), 877-888.

Haun, D. B., Rapold, C. J., Call, J., Janzen, G., \& Levinson, S. C. (2006). Cognitive cladistics and cultural override in Hominid spatial cognition. Proceedings of the National Academy of Science, 103(46), 17568-17573.

Henson, R. N. (2006). Efficient experimental design for fMRI. In K. J. Friston, J. Ashburner, S. Kiebel, T. Nichols, \& W. Penny (Eds.), Statistical parametric mapping: The analysis of functional brain images (pp. 193-210). London: Elsevier.

Iaria, G., Petrides, M., Dagher, A., Pike, B., \& Bohbot, V. D. (2003). Cognitive strategies dependent on the hippocampus and caudate nucleus in human navigation: Variability and change with practice. The Journal of Neuroscience, 23(13), 5945-5952.

Johnson-Laird, P. N. (1980). Mental models in cognitive science. Cognitive Science, 4, 71-115.

Keehner, M., Guerin, S. A., Miller, M. B., Turk, D. J., \& Hegarty, M. (2006). Modulation of neural activity by angle of rotation during imagined spatial transformations. NeuroImage, 33(1), 391-398.

Kemmerer, D. (2006). The semantics of space: Integrating linguistic typology and cognitive neuroscience. Neuropsychologia, 44(9), 1607-1621.

Kincade, J. M., Abrams, R. A., Astafiev, S. V., Shulman, G. L., \& Corbetta, M. (2005). An event-related functional magnetic resonance imaging study of voluntary and stimulus-driven orienting of attention. Journal of Neuroscience, 25(18), 4593-4604.

Lakoff, J., \& Johnson, M. (1980). Metaphors we live by. Chicago.

Leech, G., Rayson, P., \& Wilson, A. (2001). Word frequencies in written and spoken English: Based on the British National Corpus. London: Longman.

Levinson, S. C. (2003). Space in language and cognition. Cambridge: Cambridge University Press.

Levy, R., \& Goldman-Rakic, P. S. (2000). Segregation of working memory functions within the dorsolateral prefrontal cortex. Experimental Brain Research. Experimentelle Hirnforschung. Experimentation Cerebrale, 133(1), 2332.

Milner, A. D., Djickerman, H. C., \& Carey, D. P. (1999). Visuospatial processing in a pure case of visual-form agnosia. In N. Burgess, K. J. Jeffery, \& J. O'Keefe (Eds.), Spatial functions of the hippocampal formation and the parietal cortex (pp. 442-466). Oxford: Oxford University Press.

Neggers, S. F. W., Van der Lubbe, R. H. J., Ramsey, N. F., \& Postma, A. (2006). Interactions between ego- and allocentric neuronal representations of space. NeuroImage, 31(1), 320-331.

Nobre, A. C., Coull, J. T., Maquet, P., Frith, C. D., Vandenberghe, R., \& Mesulam, M. M. (2004). Orienting attention to locations in perceptual versus mental representations. Journal of Cognitive Neuroscience, 16(3), 363-373.

O'Keefe, J., \& Burgess, N. (1996). Geometric determinants of the place fields of hippocampal neurons. Nature, 381(6581), 425-428.

O'Keefe, J., \& Nadel, L. (1978). The hippocampus as a cognitive map. Oxford: Oxford University Press.

Oliveri, M., Turriziani, P., Carlesimo, G. A., Koch, G., Tomaiuolo, F., Panella, M., et al. (2001). Parieto-frontal interactions in visual-object and visualspatial working memory: Evidence from transcranial magnetic stimulation. Cerebral Cortex (New York, N.Y.: 1991), 11(7), 606-618.

Pessoa, L., Gutierrez, E., Bandettini, P. A., \& Ungerleider, L. G. (2002). Neural correlates of visual working memory: fMRI amplitude predicts task performance. Neuron, 35(5), 975-987.

Pessoa, L., Kastner, S., \& Ungerleider, L. G. (2003). Neuroimaging studies of attention: From modulation of sensory processing to top-down control. Journal of Neuroscience, 23(10), 3990-3998.

Postle, B. R., Idzikowski, C., Sala, S. D., Logie, R. H., \& Baddeley, A. D. (2006). The selective disruption of spatial working memory by eye movements. Quarterly Journal of Experimental Psychology, 59(1), 100-120. 
Rowe, J. B., Toni, I., Josephs, O., Frackowiak, R. S., \& Passingham, R. E. (2000). The prefrontal cortex: Response selection or maintenance within working memory? Science, 288(5471), 1656-1660.

Schenk, T. (2006). An allocentric rather than perceptual deficit in patient D.F. Nature Neuroscience, 9(11), 1369-1370.

Schmidt, D., Krause, B. J., Weiss, P. H., Fink, G. R., Shah, N. J., Amorim, M. A., et al. (2007). Visuospatial working memory and changes of the point of view in 3D space. NeuroImage, 36(3), 955-968.

Shallice, T. (1988). From neuropsychology to mental structure. Cambridge: Cambridge University Press.

Smith, E. E., \& Jonides, J. (1998). Neuroimaging analyses of human working memory. Proceedings of The National Academy of Sciences of The United States of America, 95(20), 12061-12068.

Talmy, L. (2000). Toward a cognitive semantics. Cambridge: MIT Press.

Ungerleider, L. G., Courtney, S. M., \& Haxby, J. V. (1998). A neural system for human visual working memory. Proceedings of The National Academy of Sciences of The United States of America, 95(3), 883-890.
Vogeley, K., May, M., Ritzl, A., Falkai, P., Zilles, K., \& Fink, G. R. (2004). Neural correlates of first-person perspective as one constituent of human self-consciousness. Journal of Cognitive Neuroscience, 16(5), 817827.

Wallentin, M., Roepstorff, A., Glover, R., \& Burgess, N. (2006). Parallel memory systems for talking about location and age in precuneus, caudate and Broca's region. NeuroImage, 32(4), 1850-1864.

Wallentin, M., Weed, E., Østergaard, L., Mouridsen, K., \& Roepstorff, A. (in press). Accessing the mental space-Linguistic and visual spatial memory processes overlap in precuneus. Human Brain Mapping, doi: $10.1002 / \mathrm{hbm} .20413$.

Wraga, M., Shephard, J. M., Church, J. A., Inati, S., \& Kosslyn, S. M. (2005) Imagined rotations of self versus objects: An fMRI study. Neuropsychologia, 43(9), 1351-1361.

Zacks, J. M., \& Michelon, P. (2005). Transformations of visuospatial images. Behavioral and Cognitive Neuroscience Reviews, 4(2), 96-118. 